\title{
Minimal impact surgical and prosthetic approach to maximize peri-implant tissue expression for the anterior aesthetic area restoration
}

\section{Luigi Canullo, DDS, PhD - Marco Tallarico, DDS - Martina Caneva, DDS - Roberto Cocchetto, MDM @ Bio-LoGIC}

Background and Aim

To prevent esthetic failures in the esthetic area, two different strategies have been proposed:

- immediate restoration of post-extraction site using bone-level implant in association to site regeneration plus connective tissue graft, or

- delayed tissue-level implant insertion in association with GBR. Presence of the IAJ at the bone level, crown overlapping, need for a supplementary surgery (immediate approach) and risk of titanium divergent implant neck exposure (delayed approach) represent drawbacks to compensate post-extraction tissue shrinkage.

The purpose of this prospective cohort study was to investigate, over a 36month period, hard and soft tissue response to a different approach for replacing missing teeth in the anterior esthetic area: transmucosal implant presenting a convergent collar inserted in regenerated post-extraction site.
Results

At the 36-month follow-up, 21 consecutive patients (9 men and 12 women with mean age of $63.7 \pm 14$ years) with 25 implants were included in this study. All implants resulted clinically osseointegrated, stable, and showed no sign of infection. At baseline, interproximal radiographs revealed no bone defect around implants. After an initial minimal bone loss $(0.09 \pm 0.144 \mathrm{~mm})$, at the last follow-up, radiographic analysis showed a stable condition of bone remodeling (mean value: $0.09 \pm 0.08$, range 0.0 to $0.5 \mathrm{~mm}$ ).

No statistically significant horizontal dimensional changes of the alveolar ridge were observed between each time points (Tab 1). Mean soft tissue levels significantly improved between baseline and $T_{3}$ timepoints. The mean height of the mesial and distal papilla changes was $-0.18 \pm 0.12$ and $-0.17 \pm$ 0.09 , respectively. The level of the labial gingival margin was $-0.11 \pm 0.063$ (Tab 2). Periodontal parameters never exceeded the physiological levels. Patient satisfaction was $9.02 \pm 0.71$

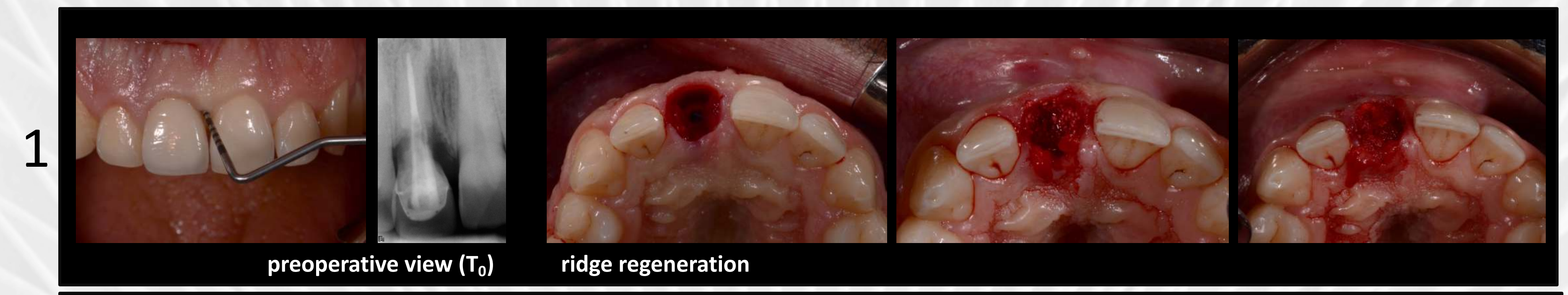

2

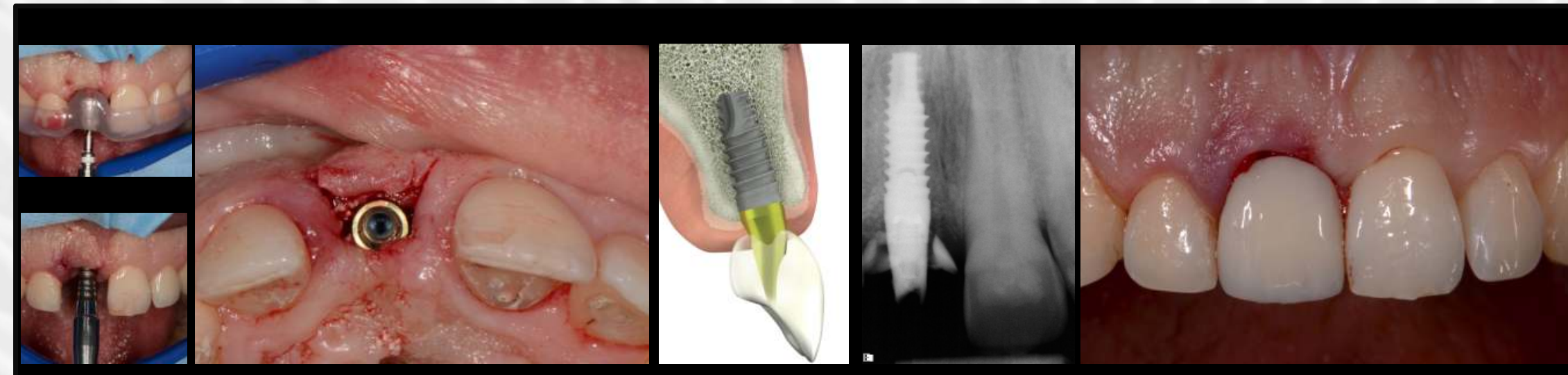

implant insertion \& immediate provisionalization

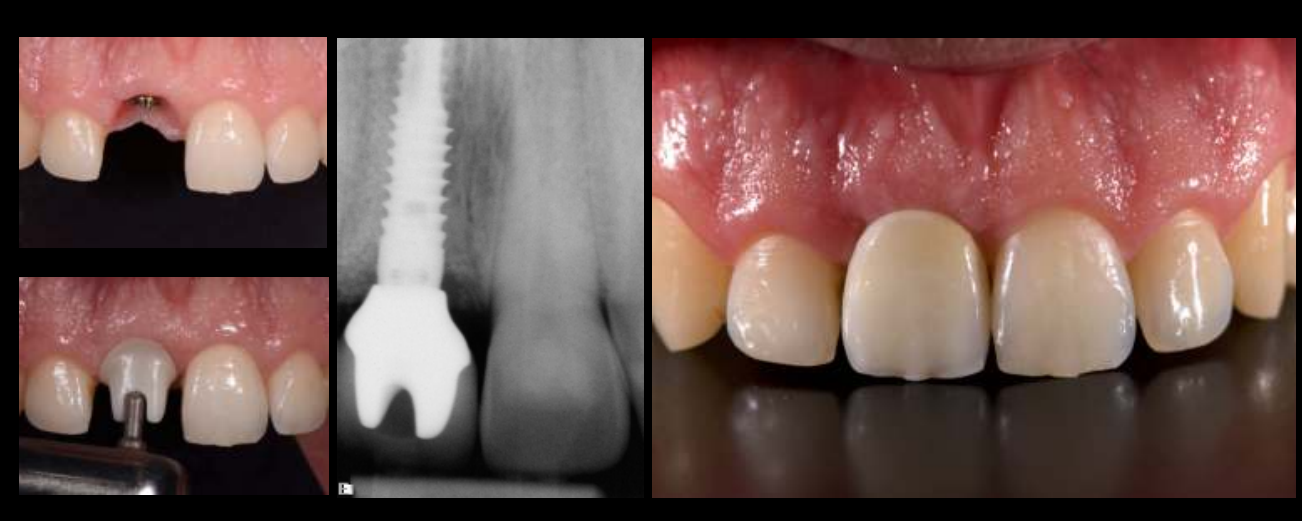

definitive restoration $\left(T_{1}\right)$
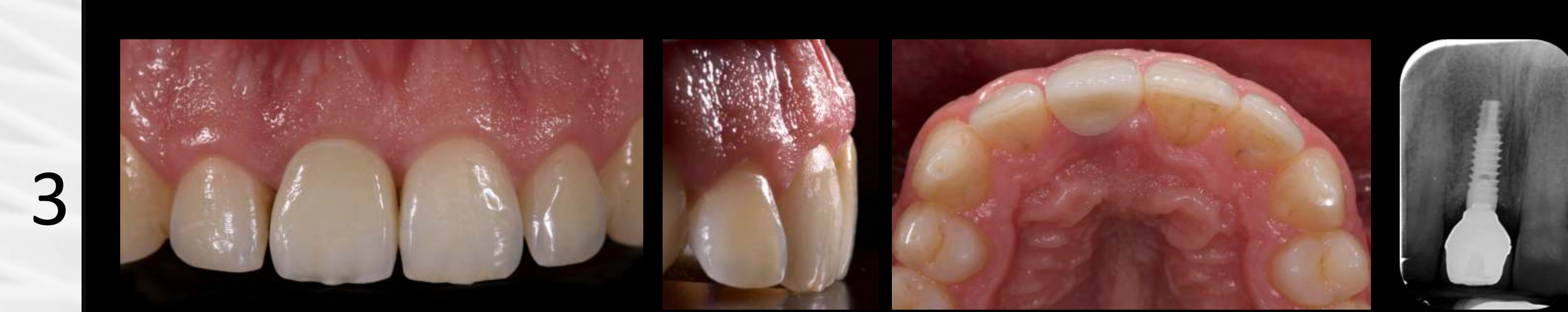

cast model super impression

3-year follow-up $\left(T_{3}\right)$

Materials \& Methods

From June 2013 to Jan 2014, all consecutive patients needing an implant supported restoration in the aesthetic anterior maxillary area between canines were enrolled. Six months after hopeless tooth extraction and alveolar socket graft with a Magnesium-enriched Hydroxyapatite (Fig 1), a transmucosal type implant with convergent walls collar was midcrestaly inserted with a semi-flapless surgery. Immediate provisional restoration was inserted. Two months thereafter, an impression was taken and a definitive zirconia abutment with a provisional restoration was positioned. Two weeks after, the definitive metal-free restoration was seated (Fig 2). Clinical parameters, pictures, radiographs and impressions were taken at the definitive restoration time point and after 6 (T1), 18 (T2) and 36 (T3) months. Using dedicated software, radiographic analysis (to detect marginal bone level changes) were performed (Fig 3).

Cast models at $T_{0}$ and $T_{3}$ were scanned and digitally superimposed to detect tissue vertical and horizontal changes.

At the end of the study, patient satisfaction, using a VAS scale, was evaluated.

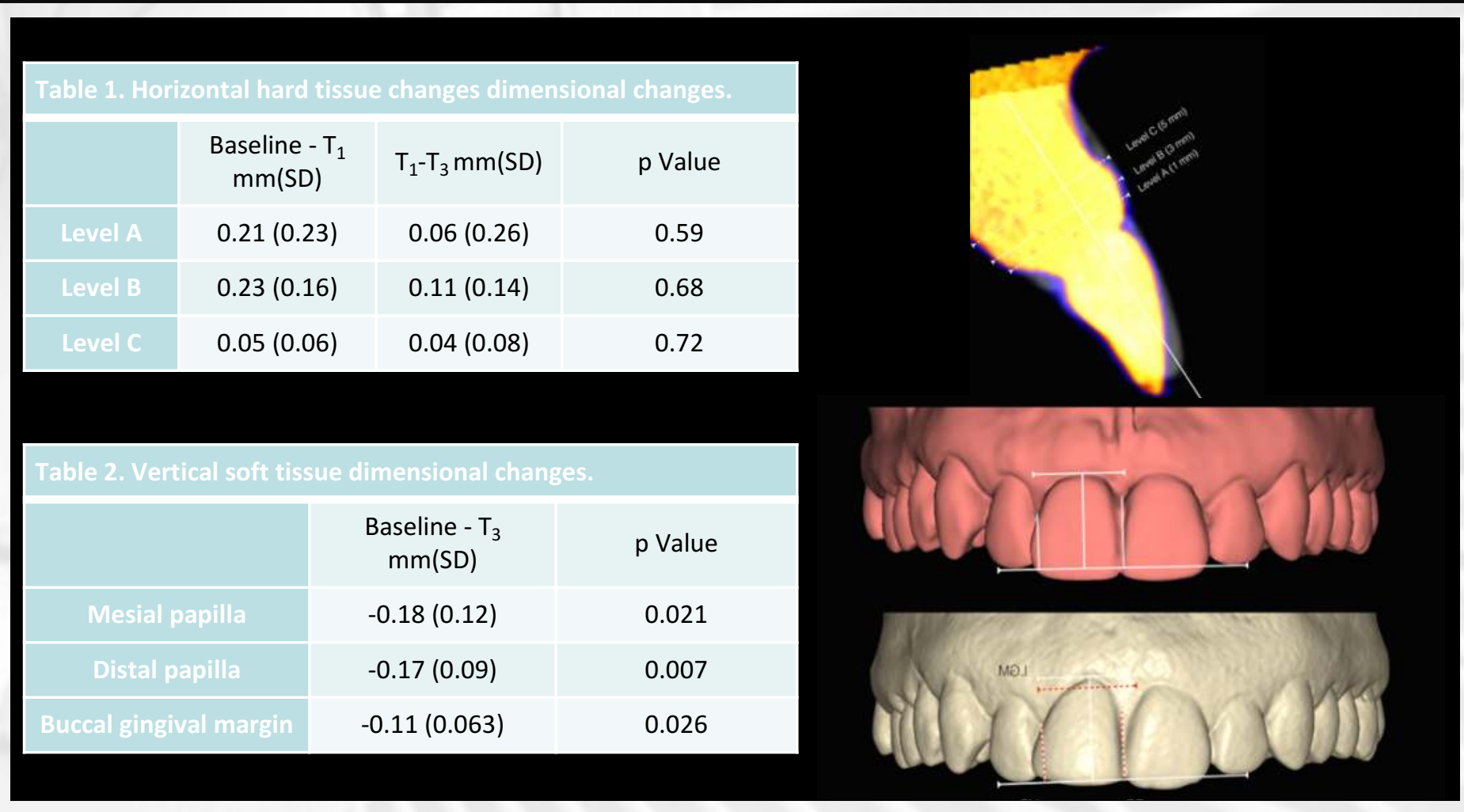

Conclusions

With the limitation of this preliminary trial, the analyzed minimal impact surgical and prosthetic approach allows to obtain encouraging results also in highly esthetically demanding cases even without any supplementary surgery. In fact, because of the insertion in a regenerated site, the absence of an AlJ at bone level and the implant peculiar convergent collar shape, the fixture can be inserted in a more proper midcrestal position. 